\title{
The Mass/Count Distinction: Evidence from On-Line Psycholinguistic Performance
}

\author{
Brendan Gillon \\ McGill University, Montreal, Quebec, Canada \\ Eva Kehayia
}

McGill University and Jewish Rehabilitation Hospital, Montreal, Quebec, Canada and Vanessa Taler McGill University, Montreal, Quebec, Canada

\begin{abstract}
Under the hypothesis that the mass/count distinction in English is marked by a monovalent lexical feature, this article investigates whether features, lexical or morphosyntactic, play a role in simple lexical decision. Research findings have yet to settle how many features are accessed during lexical decision and to what extent morphosyntactic features are computed out of context. We used two on-line lexical decision experiments (simple and morphosyntactic priming). Results show that the lexical feature "mass" is computed in both experiments. However, the morphosyntactic feature "plural" is subject to task-specific effects and surfaces only where operative. (c) 1999 Academic Press
\end{abstract}

Key Words: mass; count; singular; plural; feature; lexical access; lexical semantics.

Psycholinguistic investigations of the mental lexicon have, thus far, shown that an increased processing load, either of grammatical features encoded in the representation or of morphological operations, can affect reaction times (RTs) during word recognition in on-line psycholinguistic experiments (Taft \& Forster, 1975; Laudanna et al., 1992; Niemi et al., 1994; Kehayia \& Jarema, 1994). Little is known about the role of grammatical features in online word recognition; even less is known about such features as mass for nouns, though neurolinguistic findings have shown dissociations in the performance of patients on different noun classes, including mass and count nouns.

Address correspondence and reprint requests to Brendan Gillon, Department of Linguistics, McGill University, 1085 Avenue Docteur-Penfield. Montreal, Quebec H3A 1A7, Canada.

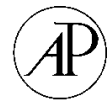


Before describing our experiments which address the mass/count distinction, we briefly outline the morphosyntactic complexity of English nouns. English has four noun classes: proper names, pronouns, mass nouns, and count nouns, the latter two comprising the class of common nouns, the exclusive concern of our study. The mass/count distinction manifests itself in English in a number of ways. For example, count nouns admit a morphological contrast between singular and plural (e.g., table and tables), whereas mass nouns do not (e.g., furniture and *furnitures). Also, cardinal numerals (e.g., two, three, etc.) and quasi-cardinal numerals (e.g., few, several) modify count nouns (e.g., two tables), never mass nouns (e.g., *two furniture).

The semantic difference between these two classes of common nouns is this. On the one hand, a singular count noun which applies to an object does not apply to any of its proper parts. For example, the word table applies to a single table, but it does not apply to any of its proper parts, say, one of its legs. In this sense, the members of the denotation of a count noun are indivisible, or atomic. On the other hand, a singular mass noun which applies to an item may apply to some, or even all, of its proper parts. The word oil, for example, applies to every mechanically obtainable portion of a puddle of oil. It is important to stress that while a mass noun which applies to an item may, as in the case of oil, apply to its proper parts, it need not. The word furniture applies to a single table, but does not apply to the table's leg. A member of the denotation of a mass noun such as oil is divisible and hence said to be nonatomic (MN), while a member of the denotation of a mass noun such as furniture is not divisible and hence said to be atomic (MA). In our study, we tracked both kinds of mass nouns.

In addition, four subclasses of common count nouns were tracked: duals (DL) such as rope, so-called because they double as count nouns and mass nouns; fossils (FS) such as man, so-called because they have suppletive plurals which are vestiges of early English; invariable plurals (IP), or pluralia tantum, such as goggles, so-called because they never lose the plural suffix -s; and plural mass (MP) nouns such as seconds, so-called because of some of the morphosyntactic properties they share with mass nouns. (See Table 1 below; see Gillon 1992 for further details.)

TABLE 1

Noun Classes Tested

\begin{tabular}{|c|c|c|}
\hline & Singular & Plural \\
\hline Regular count nouns (RC) & table & tables \\
\hline Fossils (FS) & man & men \\
\hline Invariant: -s (IP) & & trousers \\
\hline Mass: plural (MP) & & comics \\
\hline Mass: nonatomic (MN) & water & \\
\hline Mass: atomic (MA) & furniture & \\
\hline Dual (DL) & rope & ropes \\
\hline
\end{tabular}


The singular plural distinction is a morphosyntactic one. It is marked by a suffix or suppletive form and is manifested through the morphosyntactic requirements of agreement. No overt, dedicated suffix marks the mass count distinction in English; rather, it appears indirectly through the morphosyntax of grammatical number and through cooccurrence restrictions. Thus, the mass count distinction is a lexical one. However, both distinctions-mass count and singular plural-can be easily captured by features, indeed by monovalent features: briefly, mass nouns are assigned the feature " $\mathrm{M}$," whereas count nouns are not; plural nouns are assigned the feature "P,", while singular nouns are not.

\section{HYPOTHESIS}

The fundamental assumption is that during word recognition processing load increases with an increase of features to be computed. A priori, two things are clear. First, it is an open question whether features, lexical or morphosyntactic, play a role in simple lexical decision. To date, research findings are controversial as to how many features are accessed during lexical decision and to what extent morphosyntactic features are computed out of context. Second, features must play a role in the determination of acceptable combinations. Our aim is to proceed step by step so as to isolate whether and where the features come into play. To this effect we used two on-line visual lexical decision tasks. We used a simple lexical decision task to probe lexical features and a morphosyntactic priming task (henceforth called " "priming task") to probe morphosyntactic features. We anticipated that if lexical and morphosyntactic features surface during these tasks, they would have an effect on word recognition patterns.

\section{Methodology}

Experiments were run using PsyScope 1.1 (MacWhinney, 1996). Subjects were asked to indicate whether the target on the screen was a real word in English by pressing the designated YES/NO keys on the keyboard. The dependent variables were reaction time (RT) and error rate. The independent variables were: (a) stimulus type, (b) number (singular/plural), and (c) grammaticality (priming task).

\section{EXPERIMENT 1-SIMPLE LEXICAL DECISION}

\section{Subjects}

Twenty-five native speakers of English, ages 18 to 58 years, with 14 to 18 years of education, participated.

\section{Stimuli}

The total set of stimuli comprised 300 real words: 150 experimental stimuli, mass and count nouns, and 150 fillers, which were verbs in the past tense or in the present tense. There were 
also 280 nonwords created by changing the first or second phoneme of the real words, while respecting the phonotactic constraints of English.

\section{Procedure}

Stimuli were presented in lowercase. Subjects first saw a mask comprising a series of pound signs (\#\#\#\#\#) in the center of the screen, matching the number of characters of the preceding stimulus. It was presented for $200 \mathrm{~ms}$ and was followed by a pause of $150 \mathrm{~ms}$. The target appeared immediately after the pause. The interstimulus interval was set at $200 \mathrm{~ms}$. Stimuli were presented in blocks of 80 . The actual test was preceded by a practice session comprising 10 items and a pretest comprising 5 trial items.

\section{Results}

Before proceeding with the analysis, erroneous responses were isolated; they ranged from 0.4 to $3.4 \%$ across the different categories, while the IP category yielded the highest error rate, $8.7 \%$.

Two types of comparisons were conducted for the experimental items. The first compared mass and count nouns. The second contrasted singular and plural nouns for the DL, FS, and RC categories.

With respect to the first comparison, a repeated-measures ANOVA revealed a main effect of category (Mass/Count) for the singular nouns (DL, $\mathrm{FS}$, and RC), on the one hand, and MA nouns on the other $[F(1,25)=$ $11.150, p<.0001]$. A Fischer post hoc analysis also revealed a significant difference between mass and regular count nouns (MA/RC, $p<.0001$ ). (See Table 2 below.)

This finding is not surprising if we assume that processing load increases proportionally to the number of features processed and that during word recognition the lexical feature $M$ is being accessed only for mass nouns. It must be noted that frequency was not found to be a determining factor in the above difference.

Within the set of plural nouns, a repeated-measures ANOVA revealed a main effect for the category of invariant plural nouns (IP), where IP nouns were significantly slower than all others $[F(1,25)=33.495, p<.0001]$. Two factors are thought to play a role here: the low frequency of items

TABLE 2

Paired Comparisons between Singular and Plural Nouns in Simple Lexical Decision

\begin{tabular}{lll}
\hline Category & Singular & Plural \\
\hline DL (candy) & 593.4 & 597.7 \\
FS (mouse) & 592.7 & 585.7 \\
RC (baby) & 572.7 & 585.1 \\
IP (trousers) & & 666.7 \\
MA (money) & 628.5 & \\
MN (sugar) & 619.0 & 605 \\
MP (comics) & &
\end{tabular}


in the IP category as well as their susceptibility to faulty parsing during word recognition. Thus, an IP noun such as trousers is liable to be parsed as trouser-s, searched for as trouser, and, when not found, searched for as trousers. Further evidence for faulty parsing comes from the fact that IPs have the highest error rate $(8.7 \%)$ of any nominal class.

With respect to the mass/count distinction, Fischer post hoc analyses revealed a significant difference between the category of MP nouns and those of RC and FS nouns (MP/RC, $p<.0001 ; \mathrm{MP} / \mathrm{RC}, p<.0001$ ). With respect to the contrast between singular and plural within the DL, FS, and RC noun groups, repeated-measures ANOVAS revealed no significant effect. While this finding is as expected for DLs, given their dual nature, and for FSs, given their fossilized no $-s$ plural, one wonders about RC, where one may have expected significantly longer RTs if parsing of the plural marker $-s$ takes place. However, given the nature of the task, where morphosyntactic features such as ' $\mathrm{P}$ ', (plural) need not be accessed and given the high frequency of RC nouns, one may hypothesize that instead of parsing the plural $-s$, subjects opted for a whole-word access recognition process.

\section{EXPERIMENT II-LEXICAL DECISION WITH MORPHOSYNTACTIC PRIMING}

In this task, each stimulus was preceded by a determiner or adjective to yield grammatical or ungrammatical combinations, e.g., three babies-*three baby; mисh mud-*many mud. The same types of determiner primes were used across noun types. Assuming that the morphosyntactic prime activates a number of features and creates expectations for a set of specific features in the target, it is hypothesized that whenever a mismatch occurs between what the prime activates and what the target activates, RTs for the target will be slower. Nevertheless, subjects were simply requested to do a lexical decision on the target and not to judge the grammaticality of the prime target.

\section{Subjects}

Twenty-two native speakers of English, ages 18 to 58 years, with 14 to 18 years of education, participated.

\section{Stimuli}

The total set of stimuli comprised 442 real word targets: 218 experimental stimuli presented in both grammatical and ungrammatical combinations, e.g., three babies-three baby and 224 verb filler pairs, e.g., wash-washed. There were also 360 nonword targets created by changing the first or second phoneme of real words while respecting phonotactic constraints of English. Nonword targets were preceded by the same morphosyntactic real-word primes as were the real-word targets, e.g., three sabies.

\section{Procedure}

Stimuli were presented in lowercase. Primes were presented for $250 \mathrm{~ms}$. The target was presented starting at 0 and was followed by a mask, consisting of a series of pound signs 
(\#\#\#\#\#) in the center of the screen, presented for $100 \mathrm{~ms}$. The interstimulus interval was set at $200 \mathrm{~ms}$. Stimuli were in blocks of 80 with a break in between blocks. The actual test was preceded by a practice session comprising 10 items and a pretest comprising 5 trial pairs.

\section{Results}

Erroneous responses were again isolated. They ranged from 0.9 to $2.6 \%$ for the grammatical conditions and from 0.5 to $3.8 \%$ for the ungrammatical conditions across all categories with the exception of the category of IP nouns that again showed an elevated error rate of $7.3 \%$ in the grammatical condition and $8.9 \%$ in the ungrammatical condition.

Repeated-measures ANOVAS revealed a main effect for category (Mass/ Count) $[F(1,22)=15.688, p<.0001]$. A post hoc analysis revealed that, as with the simple lexical decision task, within the singular grammatical category, MA nouns were significantly slower than all others (Fischer test of significance, MA/RC, $p<.01$; MA/FS, $p<.007$; MA/DL, $p<.08$ ). Similarly to the previous experiment, the IPs were responded to significantly more slowly than all other plural nouns in the grammatical condition (Fischer test of significance, IP/RC, $p<.0001$; IP/FS, $p<.0001$; IP/DL, $p<.0002$; IP/MA, $p<.0001$ ). (See Table 3 below.)

We now turn to the comparison between grammatical and ungrammatical conditions. As expected in all cases involving a mismatch between the determiner-prime and the overt morphosyntactic feature $-s$ in the target as in the $\mathrm{RC}$ and the DL categories, ungrammatical conditions yielded significantly longer RTs (in the singular condition $F(1,22)=19.979, p<.0002$, in the plural condition $F(1,22)=14.461, p<.001)$. This pattern is reversed for the MA and the IP nouns (MAg/MAug: $F(1,22)=.9, p=$ ns; IPg/Ipug: $F(1,22)=.7, p=n s)$. In both cases, the explanation lies with facilitation arising from semantic priming. Recall that the semantic import for a noun lacking the feature $M$ (i.e., a count noun) is that its denotation is atomic, whereas the semantic import for a noun possessing the feature M (i.e., a mass noun) is that its denotation need not be atomic. Atomic mass nouns

\section{TABLE 3}

Paired Comparisons between Singular (SG) and Plural (PL) Nouns in Primed Lexical Decision

\begin{tabular}{lllll}
\hline Category & $\begin{array}{l}\text { SG } \\
(\text { Gramm.) }\end{array}$ & SG (Ungramm.) & PL (Gramm.) & PL (Ungramm.) \\
\hline DL & 634.3 & 668.2 & 640.5 & 671.1 \\
FS & 608.0 & 602.0 & 608.5 & 618.1 \\
IP & & & 738.2 & 722.6 \\
MA & 676.8 & 658.9 & 653.6 & 680.4 \\
MP & 635.1 & 650.2 & 614.2 & 643.7 \\
MN & 612.3 & 639.7 & & \\
RC & & & & \\
\hline
\end{tabular}


are nouns which are exceptional in that their denotations, being atomic, suit them for being count nouns, yet they are in fact mass nouns. We hypothesize that it is this exceptionality which accounts for their longer RTs in the primed grammatical condition. Moreover, it is precisely this exceptionality which facilitates the recognition of mass atomic nouns in the ungrammatical condition, for the plural determiner-prime requires atoms for its interpretation, which fits with the exceptional nature of the mass atomic nouns. Similarly, IP nouns are recognized more quickly in the ungrammatical condition than in the grammatical one. Once again, in the ungrammatical condition the singular determiner-prime facilitates the recognition of the word.

In conclusion, our results show that the lexical feature " $M$ " is indeed computed in both simple and primed on-line word recognition. However, the morphosyntactic feature ' $\mathrm{P}$ ' ' is subject to task-specific effects and surfaces only in the second experiment, where it is operative. Further research, using different tasks, is necessary if we are to define the level at which the grammatical features that characterize English nouns surface.

\section{REFERENCES}

Gillon, B. 1992. Towards a common semantics for English count and mass nouns. Linguistics and Philosophy, 15, 597-639.

Kehayia, E., \& Jarema, G. 1994. Morphological priming (or Prim\#ing) of inflected verb forms: A comparative study. Journal of Neurolinguistics, 2, 83-94.

Kucera, H., \& Francis, W. N. 1967. Computational analysis of present-day American English. Providence, RI: Brown Univ. Press.

Laudanna, A., Badecker, W., \& Caramazza, A. 1992. Processing inflectional and derivational morphology. Journal of Memory and Language, 31, 333-348.

Niemi, J., Laine, M., \& Tuominen, J. 1994. Cognitive morphology in Finnish: Foundations of a new model. Language and Cognitive Processes, 9, 423-446.

Taft, M., \& Forster, K. I. 1975. Lexical storage and retrieval of prefixed words. Journal of Verbal Learning and Verbal Behaviour, 14, 638-647. 